\title{
Signal enhancement of the in-plane and out-of-plane Rayleigh wave components
}

\author{
R. S. Edwards, ${ }^{\text {a) }}$ X. Jian, Y. Fan, and S. Dixon \\ University of Warwick, Department of Physics, Coventry CV4 7AL, United Kingdom
}

(Received 27 June 2005; accepted 12 September 2005; published online 1 November 2005)

\begin{abstract}
Several groups have reported an enhancement of the ultrasonic Rayleigh wave when scanning close to a surface-breaking defect in a metal sample. This enhancement may be explained as an interference effect where the waves passing directly between source and receiver interfere with those waves reflected back from the defect. We present finite element models of the predicted enhancement when approaching a defect, along with experiments performed using electromagnetic acoustic transducers sensitive to either in-plane or out-of-plane motion. A larger enhancement of the in-plane motion than the out-of-plane motion is observed and can be explained by considering ultrasonic reflections and mode conversion at the defect. (c) 2005 American Institute of Physics.
\end{abstract}

[DOI: $10.1063 / 1.2128058$ ]

An effect that has recently attracted interest is an enhancement of the Rayleigh wave at a surface-breaking defect, which may be useful in pinpointing its exact position. This has been observed by several groups scanning metal samples $^{1-6}$ using lasers and/or electromagnetic acoustic transducers (EMATs) (Refs. 7-9) to generate and detect the signal. Some researchers explain the enhancement of the outof-plane signal as an effect of changing generation conditions when a laser passes over a surface-breaking defect. ${ }^{1,2}$ Enhancement has also been explained as an interference effect between the direct and reflected Rayleigh waves ${ }^{2,5,6}$ and compared to reflection coefficient measurements. ${ }^{6,10}$ Boonsang and Dewhurst ${ }^{3,4}$ used an EMAT sensitive to in-plane motion as a detector. Enhancement of up to 2.8 times the direct signal was observed when the EMAT was very close to the defect, and explained as an effect of the interaction of an EMAT with a Rayleigh wave at a $90^{\circ}$ corner. ${ }^{3,4}$

In this letter, we describe finite element modeling (FEM) of the ultrasonic waves and seek to explain the enhancement effect observed using two EMATs to generate and detect the Rayleigh wave signal in a pitch-catch configuration. We take into consideration reflected signals and mode converted signals which may interfere with the Rayleigh wave close to a defect, and measure the in-plane and out-of-plane velocity components. When using an EMAT the pulse bandwidth, the lateral extent of the receive coil and the sharpness and orientation of the defect will significantly affect the enhancement measured. Aluminium samples with slots machined perpendicular to the sample surface are used to simulate defects.

For the FEM, we consider an aluminium half-space with a crack of depth $h$ perpendicular to the surface. At the defect, a portion of the Rayleigh waves will be reflected and mode converted longitudinal and shear waves will be generated, with the amplitudes dependent on the crack depth and angle and the pulse frequency content. In an analytical model, assumptions are made to simplify the problem and these may lead to an inability to satisfactorily explain the physical situation, and FEM offers advantages over such analytical meth-

\footnotetext{
${ }^{a)}$ Electronic mail: r.s.edwards@warwick.ac.uk
}

ods. The in-plane and out-of-plane displacement components can be expressed as ${ }^{10}$

$$
\begin{aligned}
& A_{x}=A\left[e^{-\gamma_{1} z}+\left(\frac{2 k_{2}}{k_{R}}-1\right) e^{-\gamma_{2} z}\right] e^{i k_{R} x}, \\
& A_{z}=i A\left[\frac{\gamma_{1}}{k_{R}} e^{-\gamma_{1} z}+\frac{k_{R}}{\gamma_{2}}\left(\frac{2 k_{2}}{k_{R}}-1\right) e^{-\gamma_{2} z}\right] e^{i k_{R} x},
\end{aligned}
$$

where $\gamma_{1}=\sqrt{k_{R}^{2}-k_{1}^{2}}, \gamma_{2}=\sqrt{k_{R}^{2}-k_{2}^{2}}, A$ is a constant, and $k_{1}, k_{2}$, and $k_{R}$ are the wave numbers of the longitudinal, shear, and Rayleigh waves. By using the Ray theory, it is possible to calculate the in-plane and out-of-plane velocities of the different waves; of most interest here are those of the direct and reflected Rayleigh waves and the mode converted Rayleigh to surface skimming longitudinal wave from the defect, which have arrival times that will converge as the detector gets very close to the defect. The particle displacement velocity calculations will be reported in detail in a later paper. ${ }^{11}$

EMATs are velocity sensors, and the response of the detection EMAT can be modeled considering only the behavior of the wave velocities when reflected and diffracted at the defect. Figures 1(a) and 1(b) show the B-scans produced with a $3 \mathrm{~mm}$ deep slot (with a width $1 / 50$ of the wavelength) by FEM for [Fig. 1(a)] in-plane and [Fig. 1(b)] out-of-plane motion, scanning from negative to positive distance scale. The $x$ axis gives the time scale, the $y$ axis is the distance scanned in $\mathrm{mm}$, and the amplitude of the signals are given by the brightness of the plot. The generator, set to generate wideband Rayleigh waves with a central frequency of $590 \mathrm{kHz}$ and a bandwidth of $520 \mathrm{kHz}$ at the $6 \mathrm{~dB}$ level, is held fixed, and the receiver set to scan toward the defect at $y=0$.

The labeled features are the direct $(R)$ and the reflected Rayleigh waves (RR), the mode converted Rayleigh to surface skimming longitudinal wave $(L)$ from the top of the crack, the transmitted Rayleigh wave (TR) and the transmitted mode converted longitudinal wave from the bottom of the crack (TL). When the receiver is on the opposite side of the defect to the generator, the signal is reduced due to the filtering action of the crack. ${ }^{5,6}$ The surface skimming longitudinal wave is more prominent in the in-plane velocities 


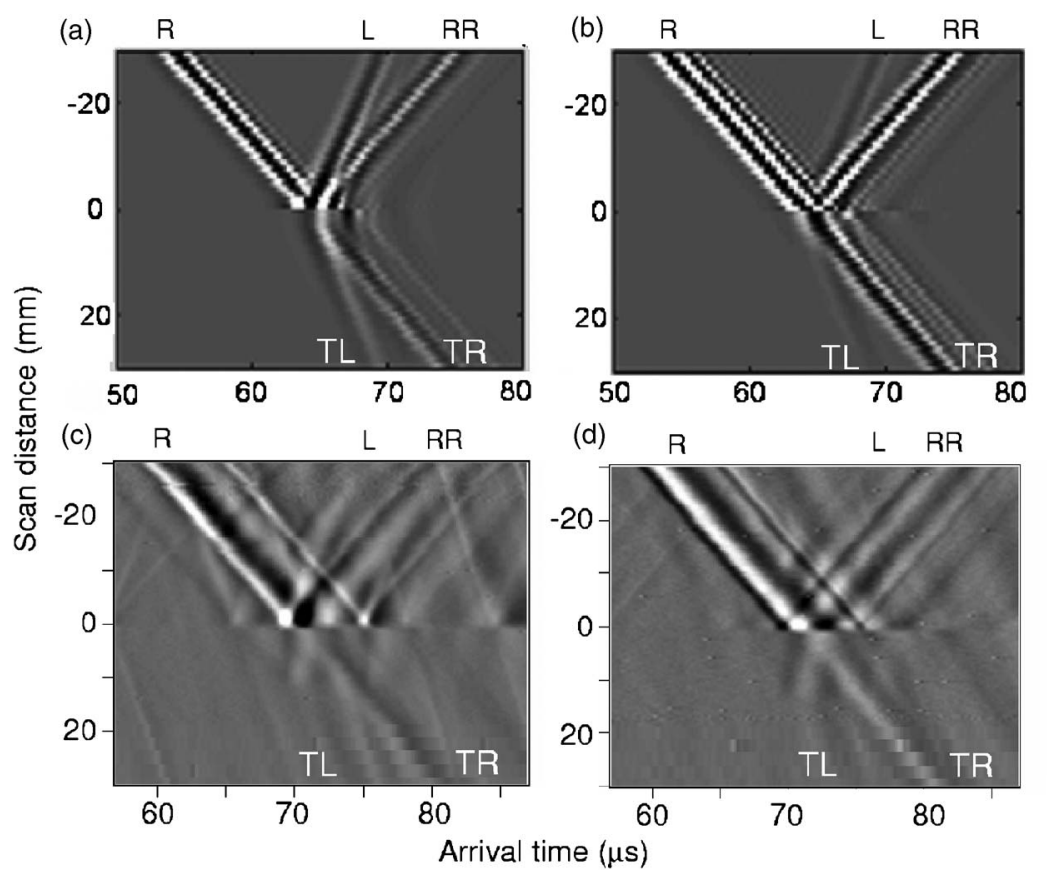

FIG. 1. B-scans from FEM for a surface crack of depth $3 \mathrm{~mm}(\approx \lambda / 2)$ for (a) in-plane and (b) out-of-plane motion; from EMAT-EMAT measurements with a $5 \mathrm{~mm}$ deep $(\approx \lambda / 3)$ machined slot with the detector sensitive to (c) in-plane, and (d) out-of-plane motion.

[Fig. 1(a)] and has a larger amplitude closer to the defect, with the amplitude decaying with distance. The amplitude of the reflected Rayleigh wave changes little over this distance scale.

Figure 3(a) shows the calculated normalized amplitude of the mode converted surface skimming longitudinal wave as a function of $x / \lambda$, where $x$ is the distance from a defect and $\lambda$ is taken as the center frequency of the Rayleigh wave, and the amplitude of the wave is negligible far from the defect. The generator here can be considered as a line source, and we consider only the two-dimensional problem. The diffracted wave will propagate into the material with the energy spread over a circumference $1 / 4 \times 2 \pi x$. Since the total energy $E$ remains constant and $E \propto$ amplitude $^{2}$, we would expect to measure an $x^{-1 / 2}$ dependence of the amplitude. ${ }^{11}$ Measurements and calculations confirm this dependence.

The basic principles of the EMATs used in these experiments have been described elsewhere. ${ }^{5-9} \mathrm{~A}$ wideband signal with a center frequency of approximately $240 \mathrm{kHz}$ is generated by a linear coil after excitation by a current pulse of duration $5 \mu \mathrm{s}$. For these experiments, we produced linear detection coils of width $0.75 \mathrm{~mm}$ and length $24 \mathrm{~mm}$ wrapped around cuboidal $\mathrm{NdFeB}$ magnets of field $0.5 \mathrm{~T}$, with the field aligned into the sample (for measuring in-plane signals) or along the sample surface (out of plane).

Figures 1(c) and 1(d) show the experimentally measured B-scans with a $5 \mathrm{~mm}$ deep $1 \mathrm{~mm}$ wide slot across the sample face $(65 \mathrm{~mm})$ for EMATs sensitive to [Fig. 1(c)] in-plane and
[Fig. 1(d)] out-of-plane motion, with the generator held fixed and the detector scanned over the defect, scanning from negative to positive distance scale. As in the model, the mode converted surface skimming longitudinal wave generated at the crack is much more prominent in the in-plane velocity measurements and its amplitude decreases with distance from the crack. Note that in Boonsang and Dewhurst, ${ }^{4}$ the wave corresponding to our $L$ wave is labeled as the reflected Rayleigh wave, ${ }^{4}$ and our reflected Rayleigh wave is labeled as a mode converted shear wave in Ref. 4 . We have confirmed our labeling by calculating arrival times for each of the waves; in particular, the slopes of the reflected Rayleigh and the mode converted shear waves should be almost equal, whereas they will be different for the reflected Rayleigh and the longitudinal surface skimming waves, as seen in the measurements. The amplitude behavior of the waves with distance as labeled here also agrees with theoretical predictions, with the reflected Rayleigh wave amplitude remaining fairly constant over the range of testing (greater than shown).

Enhancement of the signal occurs when the direct and reflected Rayleigh waves and the mode converted Rayleigh to surface skimming longitudinal wave coincide and interfere constructively (Fig. 1). The small region of enhancement is very close to the position of maximum amplitude of the surface skimming longitudinal wave, hence in the in-plane motion we would expect to see a much larger enhancement. Figure 2(a) shows the calculated signal amplitudes of our

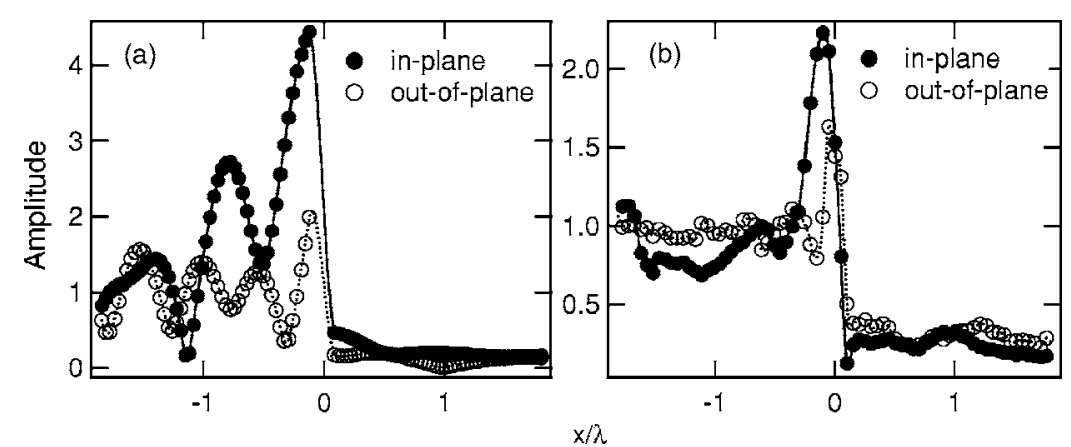

FIG. 2. Normalized amplitudes from Fig. 1 for (a) in and out-of-plane motion from FEM, (b) from experiments. 


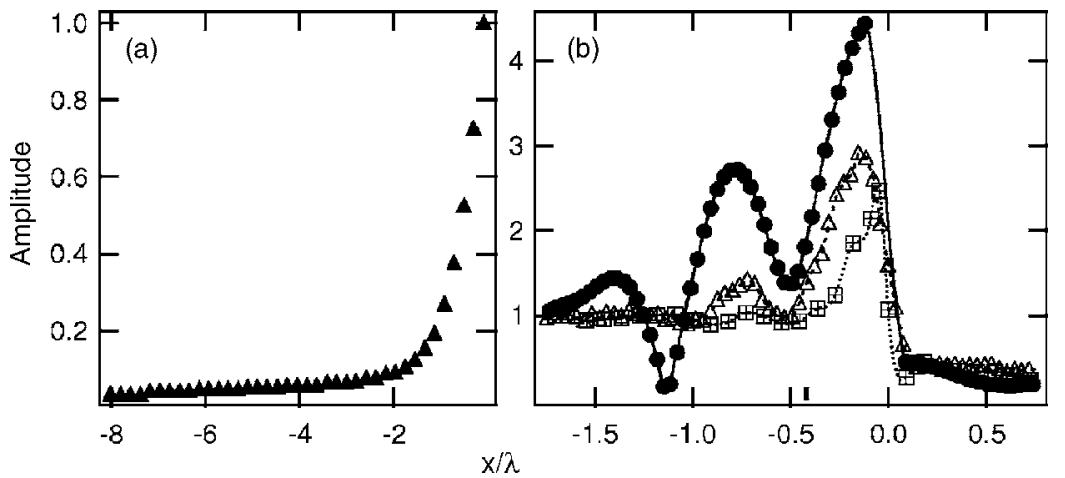

FIG. 3. (a) Normalized amplitude of the in-plane velocity of the surface skimming longitudinal wave from FEM; (b) Normalized in-plane Rayleigh wave amplitudes for experimentally measured [zero liftoff (squares) and $1.9 \mathrm{~mm}$ liftoff (triangles)] and calculations from FEM (circles). wideband Rayleigh waves for the in-plane and out-of plane velocities as a function of $x / \lambda$, normalized to the far-field signal amplitude. The signal near to the crack is enhanced, with a much larger enhancement (of approximately 5) predicted in the in-plane than the out-of-plane velocities (approximately 2.3 ).

Our earlier measurements used a wide detection coil. ${ }^{5,6}$ This will tend to average out the enhancement effect over a range of $x$ values corresponding to the coil width, and we would expect to measure a much reduced peak enhancement. A wideband pulse will also average over a range of $\lambda$. When using a narrow detection coil this averaging effect will be reduced. Figure 2(b) shows the measured normalized signal enhancements for in- and out-of-plane velocities using a $0.75 \mathrm{~mm}$ wide receive coil. The enhancement of 2.24 times the direct Rayleigh wave signal amplitude measured in the in-plane velocities, while not as high as predicted by FEM, is significantly larger than that measured in the out-of-plane velocity (1.63). The enhancement is sensitive to the crack properties, the EMAT characteristics and also the liftoff. ${ }^{12}$ To illustrate this, We show in Fig. 3(b) the measured enhancement of the in-plane signal at a liftoff for the detection EMAT of zero (squares) and $1.9 \mathrm{~mm}$ (triangles) for measurements with a fixed EMAT separation and a sharp crack, and compare it to the calculated enhancement (circles). At the higher liftoff, the measured amplitudes are closer to those predicted; in particular, note the increased amplitude of the second peak.

The enhancement of the Rayleigh wave observed when using EMATs to scan a metal sample containing a surfacebreaking crack can be explained by the interference of the direct Rayleigh wave with the reflected Rayleigh wave and the mode converted surface skimming longitudinal. The amplitude of the latter wave is only significant when close to the defect, however, it can have a very large effect on the enhancement, in particular that of the in-plane motion. The interference of this wave with the reflected Rayleigh wave close to the defect may have previously been mistaken as an extra enhancement of the reflected Rayleigh wave. Further consideration of the EMAT properties and coupling with the sample will fully explain the lift-off effect, however, the enhancement itself is sufficiently explained using the wave behavior on reflection and mode conversion. The difference between the enhancement measured in the in-plane and outof-plane signals confirms the model.

The enhancement measured will depend strongly on the lateral width of the coil and the bandwidth of the Rayleigh wave pulse, and on the sharpness of the defect. Any rounding of the corner will act to reduce the reflection coefficient and the proportion of the waves mode converted at the crack, hence reducing the enhancement. By using a sharper machined slot, in-plane enhancements of up to 2.8 have been measured. Enhancements of up to 3.1 have been measured with increased liftoff, and this level of enhancement can be explained using the FEM presented here. Measurements have also been performed on angled slots, and a similar enhancement effect is observed.

The authors thank the EPSRC for funding under Grant No. GR/S24435/01, the RCNDE, and John Reed for valuable advice.

${ }^{1}$ Z. Yan and P. B. Nagy, Rev. Prog. Quant. Nondestr. Eval. 22, 204 (2003).

${ }^{2}$ A. K. Kromine, P. A. Fomitchov, S. Krishnaswamy, and J. D. Achenbach, Mater. Eval. 58, 173 (2000).

${ }^{3}$ S. Boonsang and R. J. Dewhurst, Appl. Phys. Lett. 82, 3348 (2003).

${ }^{4}$ S. Boonsang and R. J. Dewhurst, Ultrasonics 43, 512 (2005).

${ }^{5}$ R. S. Edwards, S. Dixon, and X. Jian, J. Phys. D 37, 2291 (2004).

${ }^{6}$ R. S. Edwards, S. Dixon, and X. Jian, Rev. Prog. Quant. Nondestr. Eval. 24B, 1568 (2004).

${ }^{7}$ S. Dixon, C. Edwards, and S. B. Palmer, J. Phys. D 35, 816 (2002).

${ }^{8}$ H. M. Frost, Physical Acoustics XIV (Academic, New York, 1979), p. 179.

${ }^{9}$ S. B. Palmer and S. Dixon, Insight 45, 211 (2003).

${ }^{10}$ I. A. Viktorov, Rayleigh and Lamb Waves (Plenum, New York, 1967).

${ }^{11}$ X. Jian, S. Dixon, N. Guo, R. S. Edwards, S. T. Lock, and Y. Fan (unpublished).

${ }^{12}$ R. B. Thompson, IEEE Trans. Sonics Ultrason. 20, 340 (1973). 